ARTICLE

\title{
Surface state-induced barrierless carrier injection in quantum dot electroluminescent devices
}

\author{
Hyeonjun Lee (i) ${ }^{1}$, Byeong Guk Jeong ${ }^{2}$, Wan Ki Bae (i) ${ }^{2}$, Doh C. Lee (i) ${ }^{1 凶} \&$ Jaehoon Lim (i) ${ }^{3 凶}$
}

The past decade has witnessed remarkable progress in the device efficiency of quantum dot light-emitting diodes based on the framework of organic-inorganic hybrid device structure. The striking improvement notwithstanding, the following conundrum remains underexplored: state-of-the-art devices with seemingly unfavorable energy landscape exhibit barrierless hole injection initiated even at sub-band gap voltages. Here, we unravel that the cause of barrierless hole injection stems from the Fermi level alignment derived by the surface states. The reorganized energy landscape provides macroscopic electrostatic potential gain to promote hole injection to quantum dots. The energy level alignment surpasses the Coulombic attraction induced by a charge employed in quantum dots which adjust the local carrier injection barrier of opposite charges by a relatively small margin. Our finding elucidates how quantum dots accommodate barrierless carrier injection and paves the way to a generalized design principle for efficient electroluminescent devices employing nanocrystal emitters.

\footnotetext{
${ }^{1}$ Department of Chemical and Biomolecular Engineering, KAIST Institute for the Nanocentury, Korea Advanced Institute of Science and Technology (KAIST), Daejeon 34141, Republic of Korea. ${ }^{2}$ SKKU Advanced Institute of Nanotechnology (SAINT), Sungkyunkwan University (SKKU), Suwon, Gyeonggi-do 16419, Republic of Korea. ${ }^{3}$ Department of Energy Science, Centre for Artificial Atoms, Sungkyunkwan University (SKKU), Suwon, Gyeonggi-do 16419, Republic of Korea.凶email: dclee@kaist.edu; j.lim@skku.edu
} 
T he impetus for display devices with high color purity and low power consumption has driven intensive research on quantum dot-based light-emitting diodes (QLEDs) to exploit narrow bandwidth and high emission quantum yield (QY) of quantum dots (QDs). The QLED performance has improved hand in hand with the advances in the wet-chemical synthesis of colloidal QDs and device architectures ${ }^{1-3}$. In particular, the key to the progress in QLED efficiency is the use of organic-inorganic hybrid device architecture (denoted as hybrid QLEDs hereafter) ${ }^{4}$. It turned out that some combinations of metal oxide (e.g., $\mathrm{ZnO}$ ) electron transport layers (ETLs) and organic hole transport layers (HTLs) effectively mitigate the issue of uneven carrier injection into emissive QD layer ${ }^{5,6}$. As opposed to organic ETLs with small electron affinity $(2.0-3.0 \mathrm{eV})^{7}, \mathrm{ZnO}$ ETLs exhibit considerably large electron affinity $(\sim 4.0 \mathrm{eV})$. This contributes to balancing the electron and hole injection rates that would be impeded by a large gap between ionization energy of QD core (e.g., CdSe, CdS, or InP) and typical organic $\mathrm{HTLs}^{8,9}$.

In hybrid QLEDs, an interesting yet under-investigated question remains to be answered. There are considerable energy level differences between the conduction band minimum $(\mathrm{CBM})\left(E_{\mathrm{CBM}}\right)$ of $\mathrm{ZnO}$ and the electron state of emissive core $\left(E_{1 \mathrm{~S}_{\mathrm{e}}}\right)$ and between the hole state of emissive core $\left(E_{1 S_{\mathrm{h}}}\right)$ and the highest occupied molecular orbital (HOMO) level of the HTL ( $E_{\mathrm{HOMO}}$, HTL $)$. This means that one needs to apply external bias exceeding the optical band gap $\left(E_{\mathrm{g}}\right)$ of QDs to obtain EL from QLEDs. $E_{\mathrm{g}}, E_{1 S_{\mathrm{e}}}-E_{\mathrm{CBM}}$, ZnO, and $E_{1 S_{\mathrm{h}}}-E_{\mathrm{HOMO}, \mathrm{HTL}}$ are supposed to add up to the turn-on voltage of QLEDs (Fig. 1a) ${ }^{10}$. Interestingly, most hybrid QLEDs exhibit current density and light emission threshold voltages $\left(V_{\mathrm{J}}\right.$ and $V_{\mathrm{L}}$, respectively) comparable to or lower than $E_{\mathrm{g}} / \mathrm{e}$ despite large $E_{1 S_{\mathrm{h}}}-E_{\text {HOMO, HTL }}$ (Supplemental Table 1). The thermal effect, which is in the scale of up to $\sim 4 k \mathrm{~T} / \mathrm{e}$ or $\sim 0.1 \mathrm{~V}$, can hardly account for the sub- $E_{\mathrm{g}}$ turn-on ${ }^{10}$. It appears that $\mathrm{ZnO}$ "electron" transport materials in the hybrid QLEDs trigger barrierless "hole" injection.

Explanations for such barrierless carrier injection observed exclusively in hybrid QLEDs could include Auger-assisted upconversion $^{11}$ or thermionic emission ${ }^{12}$. The former proposes excitation of the hole by excess energy of charge transfer exciton recombining at the ETL-HTL or the QD-HTL interface. However, a large separation between ETLs and HTLs by a $10-20 \mathrm{~nm}-$ thick QD emissive layer (EML) makes the generation of chargetransfer excitons less probable. Recent QLEDs with sub- $E_{\mathrm{g}}$ turnon employing QDs with large electron confinement ${ }^{13-16}$ make it difficult to generalize this hypothesis proposed for CdSe/CdS QDs with negligible electron confinement ${ }^{5}$. Moreover, the general behavior of recent hybrid QLEDs, increasing internal quantum efficiency beyond threshold voltage and even reached to near unity, would not be validated. If it involves since the device turnon, intensifying the Auger process proportional to $n^{3}$ ( $n$ : carrier concentration) would strongly degrade internal quantum efficiency with increasing current ${ }^{17}$. On the other hand, the thermionic emission process involves the possibility of the sub- $E_{\mathrm{g}}$ emission based on a correlation between a number of thermally excited holes and current density. However, this proposed mechanism cannot provide a sufficient answer as to why only ZnO-based ETLs enable the sub- $E_{\mathrm{g}}$ operation of QLEDs while organic ETLs tend to exhibit thresholds larger than a flat band bias (Supplementary Table 1).

In addition to the limitations of these models, there is another caveat that profoundly concerns the validity of the mechanisms. In the line of reasoning, QDs and charge transport layers (CTLs) are treated as dielectrics for the simplicity of argument, perhaps to a fault. This excessive simplification is basically a result of assimilation of organic light-emitting diodes, in which work a

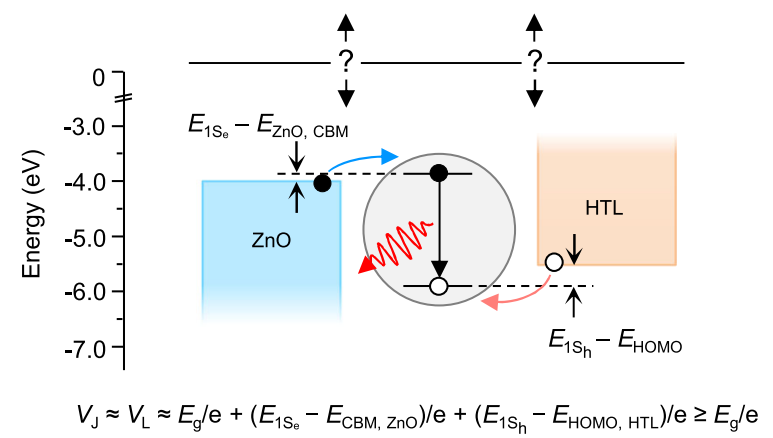

b

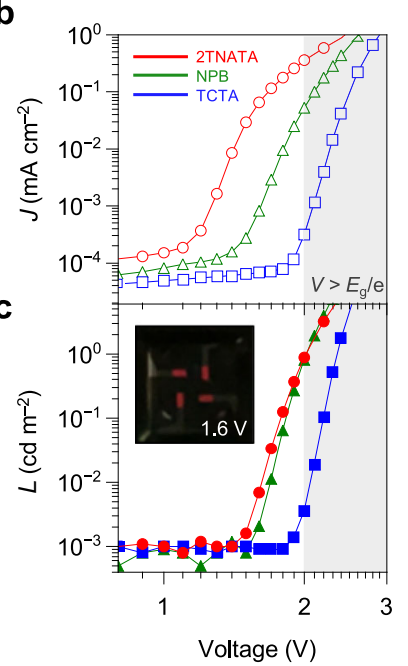

d
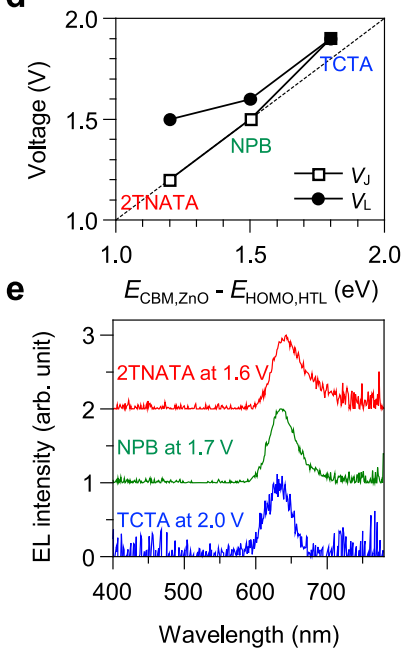

Fig. 1 Sub-band gap operation of quantum dot light-emitting diodes with energy band discontinuity. a Energy level diagram for materials used in this study without taking the interface modification into consideration. The equation estimates the current threshold $\left(V_{\jmath}\right)$ of QLEDs under the assumption that series resistance is negligible. b Current density $(J)$-voltage $(V)$ (open symbols) and c luminance ( $L$ )-voltage (closed symbols) characteristics of QLEDs employing 2TNATA (red circle), NPB (green triangle), and TCTA (blue square) HTLs. Grey shaded region indicates $V>E_{g} / e(=1.97 \mathrm{~V})$, where $E_{\mathrm{g}}$ is a bandgap of QDs. Inset shows a photographic image of 2TNATA-based QLEDs taken at an applied bias of $1.6 \mathrm{~V}$. The pixel size of devices is $1.4 \times 3.0 \mathrm{~mm}^{2} . \mathbf{d} V_{J}$ and luminance thresholds $\left(V_{\mathrm{L}}\right)$ plotted to $E_{\mathrm{CBM}}, \mathrm{ZnO}-E_{\mathrm{HOMO}}, \mathrm{HTL}$, where $E_{\mathrm{CBM}}$, ZnO is the $\mathrm{CBM}$ of $\mathrm{ZnO}$ and $E_{\mathrm{HOMO}} \mathrm{HTL}$ is a HOMO level of HTL. Dashed line indicates $V=\left(E_{\mathrm{CBM}, \mathrm{ZnO}}-E_{\mathrm{HOMO}}, \mathrm{HTL}\right) / \mathrm{e}$ for eye guidance. e EL spectra of QLEDs with different HTLs acquired at near $V_{\mathrm{L}}$.

functions $(\phi)$ of organic CTLs similarly locate at the middle of $E_{\mathrm{g}}$ and few carriers $\left(\sim 10^{10} \mathrm{~cm}^{-3} \text { or less }\right)^{18}$ are exchangeable at the junction. Core/shell QDs are almost insulating and confine carriers with wide- $E_{\mathrm{g}}$ shells, thus seemingly unable to provide carriers to be exchanged at the interface. To make sense of the barrierless carrier injection in hybrid QLEDs, however, it is necessary to consider the overall energy landscape of QLEDs including energy level equilibrium between QDs and CTLs.

In this work, we elucidate that the barrierless hole injection in hybrid QLEDs originates from the electrostatic potential gain at the QD-HTL interface. Macroscopic energy landscape is reorganized along the Fermi level of QDs derived by their surface states and this modified interfacial structure allows holes to be injected into QDs. Meanwhile, the discrete nature of individual QDs with different $E_{\mathrm{g}}$ spawns the difference in electron occupation of QDs. While the Coulombic potential of an electron in QDs to the hole is marginal compared to the extent of macroscopic potential gain, at sub- $E_{\mathrm{g}}$ condition, it modulates the 
exciton generation rate of individual QDs against nonradiative carrier loss through surface states. Poor device performance at sub- $E_{\mathrm{g}}$ condition can be translated into the dominance of trapinvolved nonradiative carrier loss through neutral wide- $E_{\mathrm{g}}$ QDs that is incapable of promoting charge injection by Coulombic attraction. We believe that our findings clarify how QDs accommodate barrierless carrier injection in hybrid QLEDs and pave the way to a generalized design principle for efficient electroluminescent devices employing nanocrystal (NC) emitters.

\section{Results and discussion}

For our study, we adopt the inverted device structure employing a $\mathrm{ZnO}$ nanoparticle (NP) ETL, InP/ZnSe/ZnS core/multishell QDs (Supplementary Fig. 1) and various organic HTLs with different ionization energies for systematic investigation: 4,4', $4^{\prime \prime}$-tris[2-naphthyl(phenyl)amino] triphenylamine (2TNATA, 5.1 eV), N,N'-di(1naphthyl)-N,N'-diphenyl-(1,1'-biphenyl)-4,4'-diamine (NPB, $5.4 \mathrm{eV}$ ), and tris(4-carbazoyl-9-ylphenyl)amine (TCTA, $5.7 \mathrm{eV})$. In current density $(J)$-voltage $(V)$-luminance $(L)$ characteristics of QLEDs, we observe an intriguing and somewhat counterintuitive trend of $V_{\mathrm{J}}$ and $V_{\mathrm{L}}$ upon using various HTLs. In the case of HTL with a larger $E_{1 \mathrm{~S}_{\mathrm{h}}}-E_{\mathrm{HOMO}}$, HTL value, both $V_{\mathrm{J}}$ and $V_{\mathrm{L}}$ are lower than what would correspond to the bandgap of QDs $\left(E_{\mathrm{g}} / \mathrm{e}=1.97 \mathrm{eV}\right.$ ) (Fig. 1b, c). For example, $V_{\mathrm{J}}=1.2 \mathrm{~V}$ and $V_{\mathrm{L}}=1.5 \mathrm{~V}$ when 2TNATA was used as a HTL. One would attribute the sub- $E_{\mathrm{g}}$ thresholds to the recombination of charge transfer complex because $V_{\mathrm{J}}$ coincides with built-in potential, $E_{\mathrm{CBM}}, \mathrm{ZnO}-\mathrm{E}_{\mathrm{HOMO}}$, HTLs (Fig. 1d). However, EL spectra acquired in the range of $V_{\mathrm{L}} \leq V \leq E_{\mathrm{g}} / \mathrm{e}$ suggest that the carriers are injected into QDs at this regime, as opposed to recombining in CTLs or via charge-transfer excitons (Fig. 1e).

To analyse the mechanism of the barrierless injection at interfaces, we probe the energy level of each component in QLEDs using ultraviolet photoelectron spectroscopy (UPS). Interestingly, InP/ZnSe/ZnS QDs on $\mathrm{ZnO}$ (ITO/ZnO/QDs) exhibit the Fermi level $\left(E_{\mathrm{F}}\right)$ at $\sim 2.6 \mathrm{eV}$ higher than the apparent $\mathrm{VBM}$ of $\mathrm{QDs}\left(E_{\mathrm{F}}-E_{\mathrm{VBM}}, \mathrm{QD}=2.6 \mathrm{eV}\right.$; Fig. $2 \mathrm{a}, \mathrm{b}$ and Supplementary Fig. 2). This value is identical when $\mathrm{Au}$ electrode was used in lieu of the ITO/ZnO substrate (Inset in Fig. 2b), suggesting the $E_{\mathrm{F}}$ pinning of QD films. To elucidate whether the $E_{\mathrm{F}}$ pinning occurs by cores or shells of the QDs, we perform a comparative study using ZnS NCs with similar size and surface chemistry (Supplementary Figs. 3 and 4 ). The $E_{\mathrm{F}}-E_{\mathrm{VBM}}, \mathrm{ZnS}$ values of $\mathrm{ZnS} \mathrm{NCs}$ on $\mathrm{ITO} / \mathrm{ZnO}$ and $\mathrm{Au}$ substrates are both $\sim 2.6 \mathrm{eV}$ (Fig. 2b and Supplementary Fig. 4). This value corresponds to the surface state emission of $\mathrm{ZnS}$ NCs centered at $2.6 \mathrm{eV}$ (Supplementary Fig. 3a) which is associated with undercoordinated $\mathrm{Zn}$ atoms on the surface ${ }^{19,20}$ leaving electronaccepting states closed to $\mathrm{CBM}^{21}$ (Inset in Supplementary Fig. 3a), not bearing on $1 S_{e}$ state of the InP core (dotted lines in Fig. 2a; see Supplementary Note 1 for details). Clear correspondence of $E_{\mathrm{F}}-E_{\mathrm{VBM}}$ between QDs and ZnS NCs excludes the involvement of In-, P-, or Se-related defect states inside QDs. Our observation clarifies that $E_{\mathrm{F}}$ and energy levels examined by UPS solely reflect characteristics of the outmost $\mathrm{ZnS}$ shell and it determines the relative energy level position of QDs to $\mathrm{ZnO}$.

The $\mathrm{ZnO}-\mathrm{QD}$ junction formation seems to accompany charge transfer to equilibrate $E_{\mathrm{F}}$. Due to a high electron concentration $\left(\sim 10^{16} \mathrm{~cm}^{-3}\right)^{22}$ and lower $\phi$ of ZnO ETL compared to QDs, electrons in $\mathrm{ZnO}$ ETL tend to migrate to adjacent electronic states of QDs, such as surface states and/or $1 \mathrm{~S}_{\mathrm{e}}$ states. The $\phi$ difference between the $\mathrm{ZnO}$ ETL and the QDs without a junction $\left(\Delta_{\phi}, \mathrm{ZnO}-\mathrm{QD}=0.35 \mathrm{eV}\right.$; see Supplementary Fig. $\left.2 \mathrm{e}\right)$ shows a disparity with observed $\Delta_{\mathrm{vac}} \mathrm{ZnO}-\mathrm{QD}$ of $0.5 \mathrm{eV}$. We attribute the offset $\Delta_{\mathrm{vac}}, \mathrm{ZnO}-\mathrm{QD}-\Delta_{\phi}, \quad \mathrm{ZnO}-\mathrm{QD}$ to the spontaneous electron charging to $1 \mathrm{~S}_{\mathrm{e}}$ states in QD EMLs. Based on the comparative study of QDs on glass, transient PL decay of ITO/ZnO/QDs taken at the entire emission range gives rise to the negative trion $\left(\mathrm{X}^{-}\right)$ decay with a fraction $\left(f_{\mathrm{e}}\right)$ of ca. $30 \%$ and the $\mathrm{X}^{-}$lifetime $\left(\tau_{\mathrm{X}^{-}}\right)$of 0.9 ns (Fig. $2 \mathrm{c}$ and Supplementary Table 2) that coincides with the $\mathrm{X}^{-}$decay lifetime extracted from photodoped $\mathrm{QDs}^{23}$ (see Methods and Supplementary Fig. 5). A modified Helmholtz equation ${ }^{24}$ yields the electrostatic potential of the partially-charged QD EML $\left(\Delta_{\mathrm{e}}\right)$ to be $\Delta_{\mathrm{e}}=0.18 \mathrm{eV}$ for $f_{\mathrm{e}}=0.3$, in close proximity with $\Delta_{\mathrm{vac}}$, $\mathrm{ZnO}-\mathrm{QD}-\Delta_{\phi}, \mathrm{ZnO}-\mathrm{QD}=0.15 \mathrm{eV}$ (Supplementary Note 2). Overall, the energy level alignment at the $\mathrm{ZnO}-\mathrm{QD}$ junction is affected not only by the $\phi$ disparity but also by the electrons injected into the QD core.

Interestingly, the surface state-derived $E_{\mathrm{F}}$ of QDs casts a profound impact on the energy landscape at the QD-HTL interface. UPS analysis on the thickness-dependent energy level of HTLs on ITO/ZnO/QDs (ITO/ZnO/QDs/HTLs) reveals that gradual band bending of $0.8-0.9 \mathrm{eV}$ develops up to $\sim 10 \mathrm{~nm}$ from the junction in the case of all tested HTLs (Supplementary Fig. 6 and Fig. 2d, e). The extent of the band bending corresponds to the $\phi$ difference between the QDs and the HTLs without junction $\left(\Delta_{\phi}\right.$, QD-HTL $=0.65-0.75 \mathrm{eV}$; see Supplementary Fig. 6$)$ on top of the contribution of negatively charged QD EMLs after deposition of HTLs $\left(\Delta_{\mathrm{e}}=0.13-0.15 \mathrm{eV}\right)$, estimated from PL decay dynamics of ITO/ZnO/QDs/HTLs (Fig. $2 \mathrm{f}$ and Supplementary Table 2). We attribute the origin of band modification to migration of thermally- or optically-generated (e.g., excitation by ambient light) electrons in HTLs to QDs; electrons are transferred to the surface states on QDs, and the remaining holes accumulate at the QD-HTL interface as space charge. We observed a minimal reduction of $f_{\mathrm{e}}$ around $3-7 \%$ point (Fig. 2f and Supplementary Table 2) using QD-selective low energy excitation at $488 \mathrm{~nm}$ (see Methods for details). The decrease in $f_{\mathrm{e}}$ attests to the recombination between electrons in $1 S_{\mathrm{e}}$ states and holes at the QD-HTL junction at equilibrium, partially allowed by the electron delocalization to surface states and a large number of holes at the interface. Summing up, the electrons seem to migrate not to the $1 \mathrm{~S}_{\mathrm{e}}$ states, but to the surface states on QDs during equilibrium.

The series of observations offer a testament that the energy level landscape in hybrid QLEDs is explained more clearly when QDs are treated as semiconductors, not as dielectrics. Building on the premise, we can make better sense of the sub- $E_{\mathrm{g}}$ turn-on in the hybrid QLEDs. First, the band bending at the QD-organic HTL junction reduces the energy level offset for the hole injection, $E_{1 S_{\mathrm{h}}}-\mathrm{E}_{\mathrm{HOMO}}$, HTL, in the way of large electrostatic potential gain. This gain effectively lowers the hole injection barrier: e.g., for 2TNATA from $0.9 \mathrm{eV}$ (non-aligned) to $\sim 0 \mathrm{eV}$, as manifested from $\sim 0.1 \mathrm{eV}$ from UPS analysis (red arrows in Fig. 2e). Second, the electron injection barrier, $E_{1 \mathrm{~S}_{\mathrm{e}}}-E_{\mathrm{CBM}} \mathrm{ZnO}$, is raised as a result of the $E_{\mathrm{F}}$ alignment, yet the increase is marginal since $E_{\mathrm{F}}$ difference is merely $0.35 \mathrm{eV}$. That is attributed to that both the $\mathrm{ZnO}$ ETL and the $\mathrm{Zn}$ chalcogenide shell have defect states derived by undercoordinated $\mathrm{Zn}$ atoms on their surface ${ }^{25,26}$. In addition, the high electron concentration of $\mathrm{ZnO}$ narrows the electron injection barrier width ${ }^{27}$. In the hybrid QLEDs examined in this work, overall electrostatic potential gain at the ZnO-QD-2TNATA interfaces (i.e., $\Delta_{\text {vac, }} \mathrm{ZnO}$-QD $+\Delta_{\text {vac, QD-HTL }}$ ) effectively lowers the total energy barrier for carrier injection into QDs by $\sim 0.4 \mathrm{eV}$, which agrees with the offset between $V_{\mathrm{L}}$, 2TNATA and $E_{\mathrm{g}} / \mathrm{e}$ of QDs. As most QDs studied in previous reports feature the zinc chalcogenide shell, the theory of band bending and $E_{\mathrm{F}}$ pinning could offer hindsight for the sub- $E_{\mathrm{g}}$ operation in hybrid QLEDs.

The explanation extends to the limitation of all-organic QLEDs with $V_{\mathrm{L}}>E_{\mathrm{g}} / \mathrm{e}$. At the QD-organic ETL junctions, one would estimate the energy barrier for electron injection to be large due 

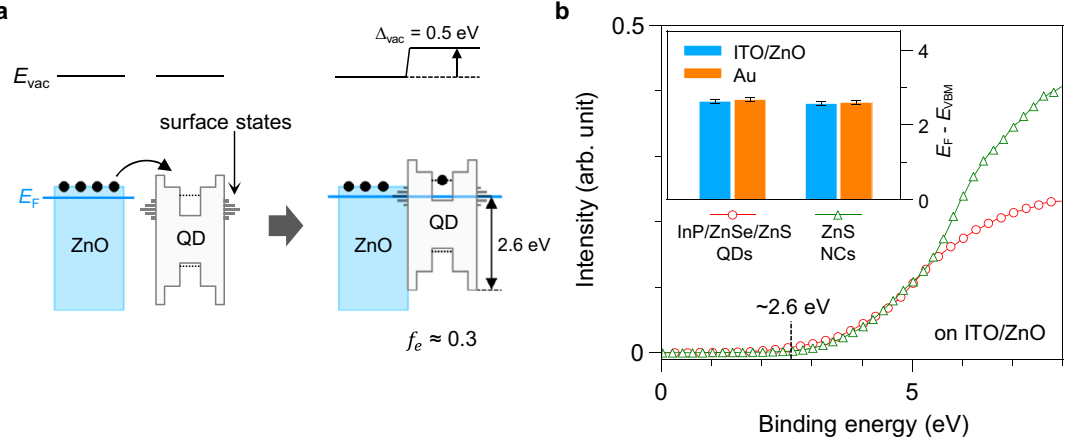

d

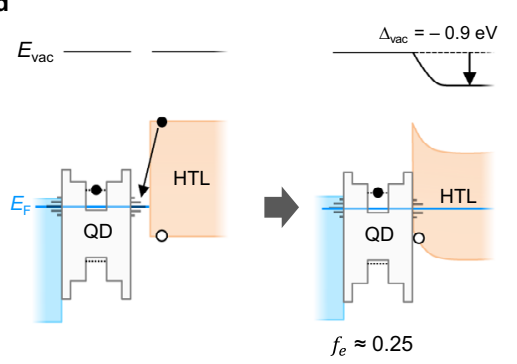

e

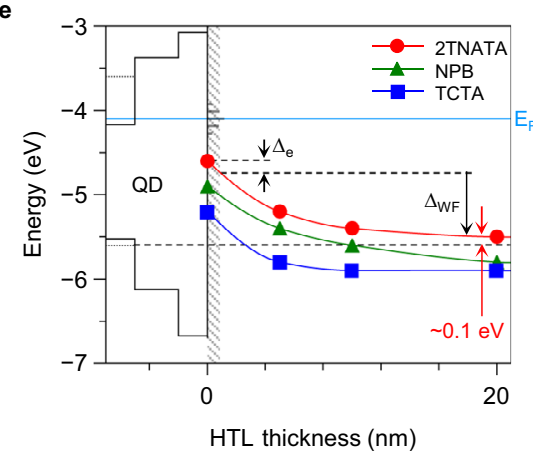

c

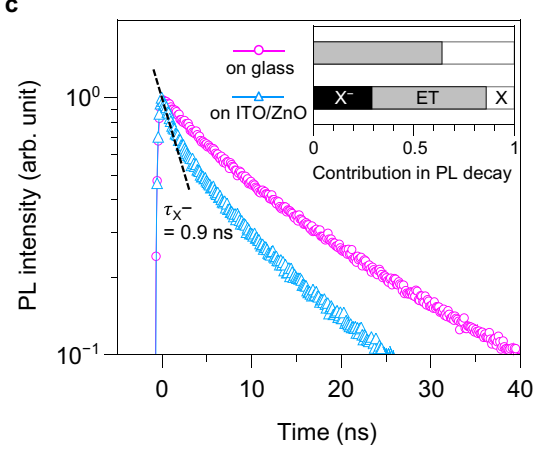

f

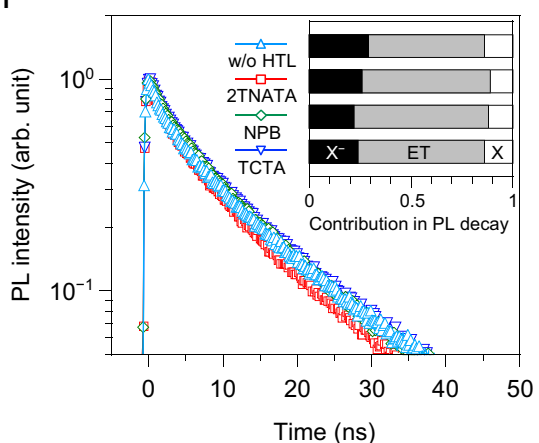

Fig. 2 Interfacial electrostatic potential gain by surface state-derived Fermi level alignment. a Modification of energy levels at a ZnO ETL-QD EML junction. Blue solid line represents Fermi level $\left(E_{\mathrm{F}}\right)$. Vacuum level shift $\left(\Delta_{\mathrm{vac}}\right)$ is estimated to be $0.5 \mathrm{eV}$. b UPS spectra of InP/ZnSe/ZnS QDs (red square) and $\mathrm{ZnS} \mathrm{NCs} \mathrm{(green} \mathrm{triangle)} \mathrm{on} \mathrm{ITO/ZnO} \mathrm{substrates} \mathrm{at} \mathrm{low} \mathrm{binding} \mathrm{energy} \mathrm{region.} \mathrm{Inset} \mathrm{shows} \mathrm{the} E_{\mathrm{F}}-E_{\mathrm{VBM}}$ values on ITO/ZnO (blue) and Au (orange) substrates. Error bars indicate an instrument resolution, $0.05 \mathrm{eV}$. c PL decay dynamics of QDs on glass (pink circle) and ITO/ZnO substrates (sky blue triangle). $\mathrm{X}^{-}$decay occurs at an early stage in the case of ITO/ZnO, with a negative trion lifetime $\tau_{\mathrm{X}^{-}}=0.9 \mathrm{~ns}$. Inset displays fractions of decay channels: $X^{-}$(black), neutral exciton (X, white), and delayed recombination by energy transfer (ET, grey). $\mathbf{d}$ Band bending at the QD-HTL junction with $\Delta_{\mathrm{vac}}=-0.9 \mathrm{eV}$. e HOMO levels of 2TNATA (red circle), NPB (green triangle), and TCTA (blue square) on ITO/ZnO/QDs substrates as a function of HTL thickness. f PL decays of QDs in ITO/ZnO/QDs/HTLs: without HTLs (sky blue triangle), 2TNATA (red square), NPB (green diamond), and TCTA (blue inverse triangle). Inset shows the contribution of $X^{-}$(black), $X$ (white), and ET (grey). All PL decays were acquired at the entire emission range with excitation at $488 \mathrm{~nm}$.

to the $\phi$ difference: for instance, $\sim 0.45 \mathrm{eV}$ for 3,5-tri(phenyl-2benzimi-dazolyl)-benzene $(\mathrm{TPBi})^{28}$ or $\sim 0.75 \mathrm{eV}$ for tris- $(8$ hydroxyquinoline)aluminium $\left(\mathrm{Alq}_{3}\right)^{29}$. The organic ETLs are likely to impose a large and wide electron injection barrier at the QD-organic ETL junction due to low carrier concentration $\left(\sim 10^{10} \mathrm{~cm}^{-3} \text { or less }\right)^{18}$, consequently resulting in increased operation thresholds (Supplementary Table 1).

Despite the closeness of electrostatic potential gain from HTLs, $V_{\mathrm{L}}$ is larger than $V_{\mathrm{J}}$ in the hybrid QLEDs with 2TNATA and NPB, while $V_{\mathrm{L}}$ and $V_{\mathrm{J}}$ are in similarly close proximity to $E_{\mathrm{g}} / \mathrm{e}$ in TCTA-based devices (Fig. 1d). To rationalize such observation, we perform the capacitance $(C)$-voltage $(V)$ analysis for 2TNATA (Fig. 3a-d) and TCTA-based QLEDs (Fig. 3e-h). We note that the increase in capacitance and energy band deformation up to $V \approx V_{\mathrm{J}}$ occurs mainly in organic HTLs. The $\mathrm{ZnO}$ ETL remains flat nearly up to the threshold voltage ${ }^{30}$ due likely to its high dielectric constant (relative dielectric constant: $\sim 8)^{31}$ and carrier concentration $\left(\sim 10^{16} \mathrm{~cm}^{-3}\right)^{22}$. The applied bias of $1.1 \mathrm{~V}$ for 2TNATA and $2.6 \mathrm{~V}$ for TCTA at peak capacitances represent a flat band condition of HTLs (Fig. $3 c$, h for respective HTL) $)^{32}$.

We found that the thresholds of 2TNATA-based QLEDs take place as holes accumulate at the QD-HTL interface. Based on the following experimental findings, we attribute the threshold behavior of 2TNATA-based QLEDs to the influence of surface defects (Fig. 3c). First, a hybrid QLED employing ZnS NCs exhibits a trap-filled limit voltage $\left(V_{\mathrm{TFL}}\right)$ at $1.0 \mathrm{~V}$ (Supplementary Fig. 7a). Second, a $L-J$ characteristic around the $V_{\mathrm{L}, 2 \mathrm{TNATA}}$ shows a super-linear behavior with a slope of $\sim 2.3$ (Supplementary Fig. 7b), suggesting the involvement of the surface states ${ }^{33}$, not the Auger recombination of carriers $\left(L \propto J^{2 / 3}\right)^{34}$ and corresponding energy up-conversion ${ }^{11,35}$. Thus, trap-involved recombination is initiated at $V=V_{\mathrm{J}, 2 \mathrm{TNATA}}$ and competes with the hole injection into QDs despite at $V \geq V_{\mathrm{L}, 2 \mathrm{TNATA}}$ (Fig. 3d). While the potential gain at the QD-2TNATA of $0.9 \mathrm{eV}$ allows for hole injection at $V \geq V_{\mathrm{L}, \text { 2TNATA }}$, the leakage current exceeds the exciton recombination current by $V=\sim 3 \mathrm{~V}$ (Supplementary Fig. 7b). We rule out multiexcitons in this regime since $J$ is too low compared to exciton recombination rate . $^{36}$.

On the other hand, for both $V_{\mathrm{J}}$, TCTA and $V_{\mathrm{L}}$, TCTA of TCTAbased QLEDs are $\sim 1.9 \mathrm{~V}$, consistent with the onset of capacitance increase (Fig. 3e). While the potential does not lead to a flat band of TCTA, the bias exceeding the built-in potential of given devices $\left(E_{\mathrm{CBM}, \mathrm{ZnO}}-E_{\mathrm{HOMO}, \mathrm{HTLs}}=1.8 \mathrm{eV}\right)$ would result in the migration of thermally generated holes to the $1 S_{h}$ state without considerable hole accumulation at the interface (Fig. 3e, g). Almost linear $L-J$ relationship above $J=10^{-2} \mathrm{~mA} \mathrm{~cm}^{-2}$ (Supplementary Fig. 7b) and $V_{\text {TFL }}$ of a device with $\mathrm{ZnS}$ QDs as large as $2.3 \mathrm{~V}$ (Supplementary Fig. 7a) suggest that the carrier leakage via traps has little to do with $J$, and hence nearly identical $V_{\mathrm{J}}$, тCTA and $V_{\mathrm{L}}$, тстA. While holes accumulate at the junction at increased $V$, the accelerated hole injection driven by sufficient electrostatic potential (dashed lines in Fig. $3 \mathrm{~h}, E_{1 \mathrm{~S}_{\mathrm{h}}}-\mathrm{E}_{\mathrm{HOMO}}$, тСтA $=-0.3 \mathrm{eV}$ ) lowers the concentration of holes at the interface and reduces trap-assisted leakage. 
a
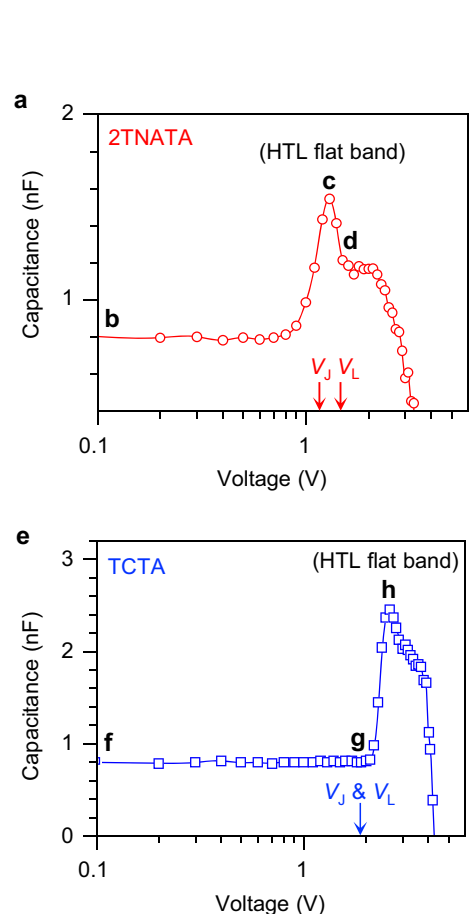
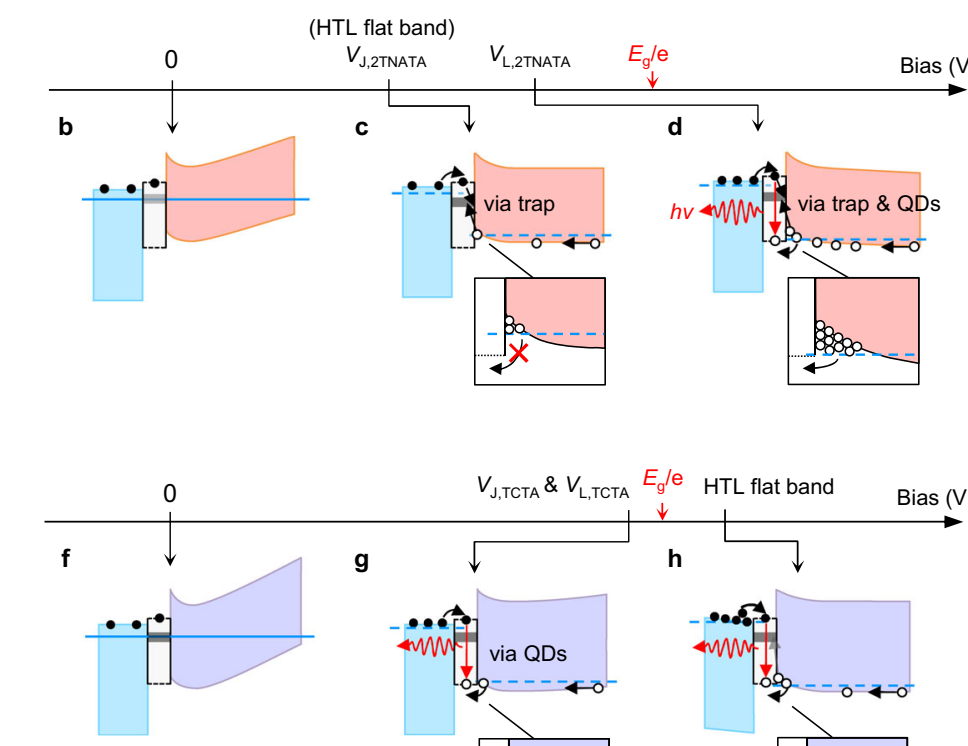

.

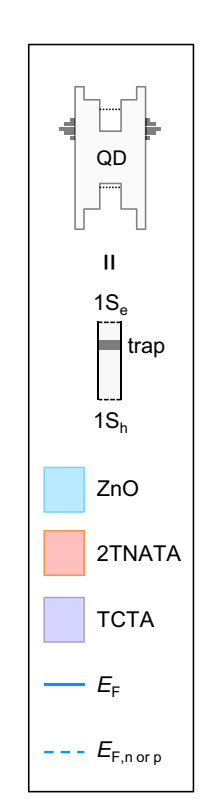

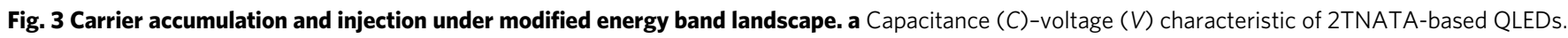
Current $\left(V_{\mathrm{J}}\right)$ and luminance thresholds $\left(V_{\mathrm{L}}\right)$ are $1.2 \mathrm{eV}$ and $1.5 \mathrm{eV}$, respectively (red arrows). b-d Band diagrams of ZnO/QD/2TNATA with increasing bias, from $V=0 \mathrm{~V}(\mathbf{b})$, to $1.2 \mathrm{~V}(\mathbf{c})$, and to $1.5 \mathrm{~V}(\mathbf{d})$. e A $C-V$ characteristic of TCTA-based QLEDs. $V_{\mathrm{J}}$ and $V_{\mathrm{L}}$ are identical to 1.9 eV (blue arrow). f-h Band diagrams of $\mathrm{ZnO} / \mathrm{QD} / \mathrm{TCTA}$ with increasing bias, from $V=0 \mathrm{~V}(\mathbf{f})$, to $1.9 \mathrm{~V}(\mathbf{g})$, to $2.6 \mathrm{~V}$ (h). Legend for $\mathbf{b}-\mathbf{d}$ and $\mathbf{f}-\mathbf{h}$ is shown on the right panel. Blue lines represent the $E_{\mathrm{F}}$ at equilibrium (solid line) and the quasi-Fermi level of electron $\left(E_{\mathrm{F}, \mathrm{n}}\right)$ and hole ( $E_{\mathrm{F}, \mathrm{p}}$ ) under applied bias (dashed line). We note that the quasi- $E_{\mathrm{F}}$ of HTLs is defined by molybdenum oxide/Al anode, assign at $\sim 0.3 \mathrm{eV}$ smaller than $E_{\mathrm{HOMO}}$, HTL.

The intervention of the trap states in carrier injection to QDs necessarily results in degradation of device performance because it lowers charge injection efficiency $\left(\eta_{\text {inj }}\right)$ in the equation of external quantum efficiency (EQE): $\eta_{\mathrm{EQE}}=\eta_{\mathrm{QY} \text {, EML }} \times \eta_{\text {inj }} \times \eta_{\text {out }}$, where $\eta_{\mathrm{QY}}$, EML is a PL QY of QD EMLs, $\eta_{\text {inj }}$ is a carrier injection efficiency, and $\eta_{\text {out }}$ is an outcoupling efficiency. Assuming that only neutral QDs are emissive $\left[\eta_{\mathrm{QY}}\right.$, EML $=\eta_{\mathrm{QY}}$, film $\times\left(1-f_{\mathrm{e}}\right)$, where $\eta_{\mathrm{QY}}$, film $=0.41$ and $f_{\mathrm{e}}=\sim 0.3$ at peak $\mathrm{EQE}$ condition; see Supplementary Fig. $8 \mathrm{a}, \mathrm{b}]$ and an outcoupling efficiency is $\sim 0.2^{8}$, the theoretical maximum EQE of QLEDs is $\sim 5.7 \%$ when $\eta_{\mathrm{inj}}=1$. An EQE curve of TCTA-based QLEDs ranging at 5\% in Supplementary Fig. 8c concludes the suppressed carrier loss via trap states and corresponding high $\eta_{\text {inj }}=\sim 0.88$. In the case of 2TNATA-based QLEDs, on the contrary, the trap-related carrier loss is initiated even before the device turn-on, consequently, the $\mathrm{EQE}$ becomes as low as $\sim 2 \%$ due to poor $\eta_{\mathrm{inj}}=\sim 0.35$.

Macroscopic investigation on the energy landscape and charge accumulation in this study provides a clear understanding on the energetics of carriers in hybrid QLEDs, how the carrier injection barrier can be relaxed by the electrostatic potential gain at the junctions. In fact, this macroscopic postulation has paved the way for various approaches promoting balanced carrier injection to QD EMLs, for instance, controlling confinement barriers 8,37 by the shell that is demonstrated in typical Cd-based QLEDs. The line of logic is based on the notion that the QD EMLs have continuum emissive states in which each state is commutable and carrier injection rates reflect average carrier occupation of entire emissive states, as is the case in conventional inorganic and organic EL devices. However, in the case of QD EMLs, the wide$E_{\mathrm{g}}$ shell and surface ligands isolate the energy states of cores, which impede the migration of charges in the planar direction. A complication of carrier injection processes arisen from the discrete nature of each QD is a main subject of the following discussion.

The electron charging of QDs on ITO/ZnO substrates in QLEDs turns out to depend on $E_{\mathrm{g}}$ of QDs, which highlights the effect of variation of individual QDs in the EML. The opposite tendency observed in spectrally-resolved PL decay ${ }^{38}$ of narrow- $E_{\mathrm{g}}$ QDs on ITO/ZnO and glass substrates suggests selective electron transfer to narrower- $E_{\mathrm{g}} \mathrm{QDs}$ thanks to lower electron injection barrier (Fig. 4a). For example, ITO/ZnO/QDs develops a fast decay pathway with a lifetime of $0.9 \mathrm{~ns}$ at $1.85 \mathrm{eV}$ (A red arrow in Inset of Fig. 4a and Supplementary Fig. 9) corresponding to $\tau_{\mathrm{X}^{-}}$ (Supplementary Fig. 5) while the glass/QDs exhibit a longer lifetime due to resonant energy transfer (ET). The $E_{\mathrm{g}}$ variation of QDs makes a marginal impact on $E_{1 \mathrm{~S}_{\mathrm{h}}}$ because of the considerably heavier effective mass of a hole $\left(m_{\mathrm{h}}{ }^{*}\right)$ than that of an electron $\left(m_{\mathrm{e}}{ }^{*}\right)$ in InP $\left(7.5 m_{\mathrm{e}}{ }^{*} \approx m_{\mathrm{h}}{ }^{*} \text {; see Supplementary Fig. } 2 \mathrm{e}\right)^{39}$. The variation of $1 S_{\mathrm{h}}$ state between the narrowest and widest- $E_{\mathrm{g}} \mathrm{QDs}$ is merely up to $\sim 41 \mathrm{meV}$ for a given QD ensemble.

Under applied bias $V \approx V_{\mathrm{L}}$, we indeed observe asymmetric and red-shifted emission profiles from 2TNATA-based QLEDs, seemingly reflecting dominant participation of narrow- $E_{\mathrm{g}}$ QDs in EL (Inset in Fig. 4b). However, the EL spectra from TCTA-based QLEDs appear unaltered from the PL spectra of QD EML (inset in Fig. $4 \mathrm{c}$ and Supplementary Fig. 10a). To make sense of the skewed EL spectra in the case of the 2TNATA-based device, we introduce the continuum density of states (DOS) model that translates the EL spectrum as recombination of electrons in the broad $1 \mathrm{~S}_{\mathrm{e}} \mathrm{DOS}$ with holes in the narrow $1 \mathrm{~S}_{\mathrm{h}}$ DOS with identical electron and hole injection rates (Fig. 4d; see Supplementary Note 3). As shown in Supplementary Fig. 10b, the theoretical results corroborate the EL spectrum of 2TNATA devices at $V \approx V_{\mathrm{L}}$ with an offset of overall potential gain. However, this model fails to account for the $V$ - 
a

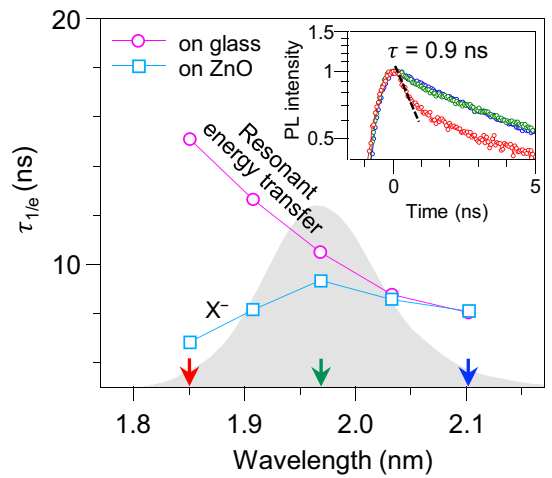

b

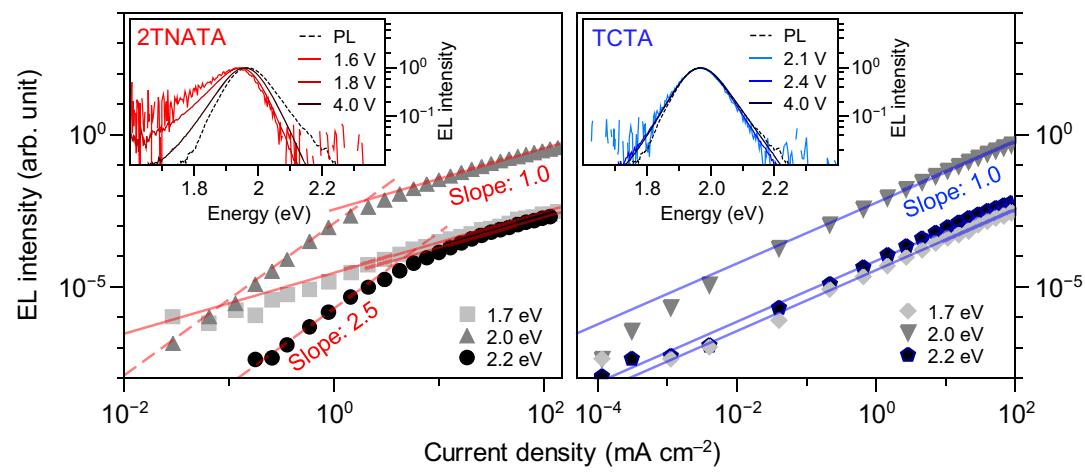

d

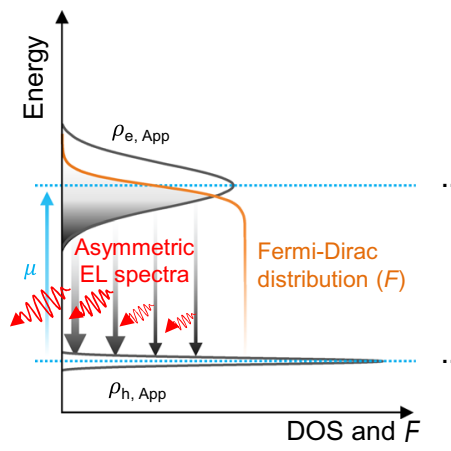

Continuum model

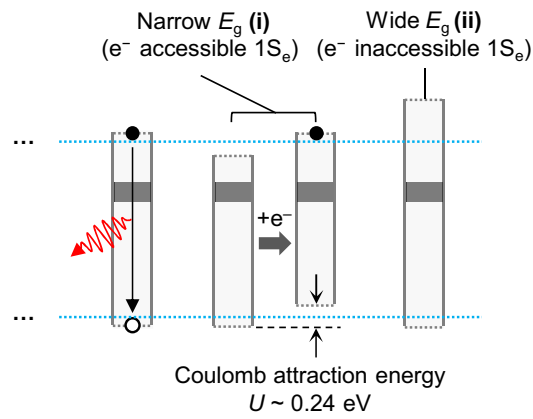

Discrete states model (at $V \approx V_{\mathrm{L}, \text { 2TNATA }}$ )

(i) Hole injection dominant to $1 \mathrm{~S}_{\mathrm{h}}$ and correlated injection

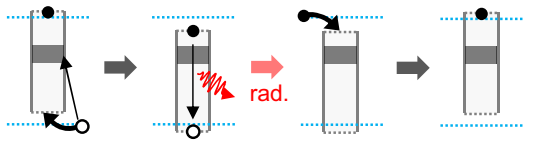

Faster $\mathrm{h}^{+}$injection to $1 \mathrm{~S}_{\mathrm{h}}$ than to surface traps

(ii) Competition between surface trapping \& injection to $1 S_{h}$

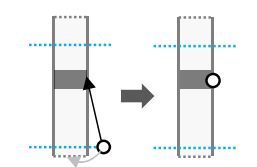

Surface trap-involved nonradiative recombination

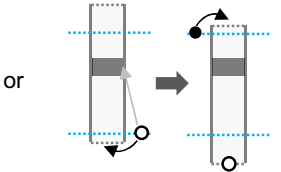

Correlated injection

Fig. 4 Local electrostatic potential gain granted by a charge in individual QDs and discrete nature of QD emissive layer. a Spectrally resolved PL decay lifetime $\left(\tau_{1 / \mathrm{e}}\right)$ of glass/QDs (magenta circle) and ITO/ZnO/QD (sky blue square) at different wavelengths. PL spectrum of QD film is provided as grey background. Inset shows PL decay traces of ITO/ZnO/QDs at 2.10, 1.97, and $1.85 \mathrm{eV}$, indicated using blue, green, and red arrows, respectively, on the $x$-axis of the main panel. $X^{-}$decay is clearly observed at $1.85 \mathrm{eV}$ with a lifetime of $0.9 \mathrm{~ns}$ in early stage (red). b, $\mathbf{c}$ EL intensity-J dependence probed at $1.7 \mathrm{eV}$ (light grey square), $2.0 \mathrm{eV}$ (dark grey triangle), and $2.2 \mathrm{eV}$ (black circle) for 2TNATA-based (b) and TCTA-based QLEDs (c). Dashed and solid lines indicate regression lines with a slope of 2.5 and 1.0, respectively. Insets show EL spectra acquired at different biases. d Schematic illustration on continuum density of state (DOS) model. At a specific electron chemical potential ( $\mu$, sky blue line), electrons occupy the apparent $1 \mathrm{~S}_{\mathrm{e}} \mathrm{DOS}\left(\rho_{\mathrm{e}}\right.$, App $)$ from the lowest state following the Fermi-Dirac distribution $(F), F \times \rho_{\mathrm{e}}$, App. On the other hand, holes in the apparent $1 \mathrm{~S}_{\mathrm{h}} \mathrm{DOS}\left(\rho_{\mathrm{h}}\right.$, App $)$ possess minimal energy distribution due to the narrowness of $\rho_{\mathrm{h}}$, App. Spectral shape is therefore determined by the distribution of $F \times \rho_{\mathrm{e}}$, App in energy scale that shows low-energy tail. e In the microscopic viewpoint, individual QDs experience different electron occupation, and carrier injection events. In 2TNATA-based QLEDs, at $V \approx V_{L}$, prior injection of an electron into narrow- $E_{g}$ QDs results in prompt injection of a hole via Coulombic attraction $(U)\left[(i)\right.$, upper right]. In the case of wide- $E_{g}$ QDs, a hole is likely to migrate to $1 S_{h}$ state or to surface states. Barring the unlikely event that the hole introduced to $1 S_{h}$ state electrostatically attracts an electron, the trap-involved nonradiative recombination is dominant.

dependent EL peak energies and spectral shapes in the case of 2TNATA-based devices. According to the model, $V \geq 2 \mathrm{~V}$ is sufficient to inject electrons to wide- $E_{\mathrm{g}}$ QDs, contradicting the observed asymmetric and red-shifted EL. The experimental results speak volumes for the effect of individual characteristics of QDs; the use of specific HTLs gives rise to the substantial differences between QDs in carrier injection rates, charge occupations, and exciton generation rates.

The spectrally resolved EL intensity from QLEDs recorded at various $J$ offers a deeper understanding of the mechanism of the carrier injection into QDs. For 2TNATA- and TCTAbased devices, we investigate the EL intensity- $J$ relationship for narrow-, intermediate-, and wide- $E_{\mathrm{g}}$ QDs with emission energies $E_{\text {emission }}=1.7,2.0$, and $2.2 \mathrm{eV}$, respectively (Fig. $4 \mathrm{~b}, \mathrm{c}$ ). The EL intensity in the 2TNATA-based QLEDs exhibits a transition from super-linear (slope: 2.5 ) to linear $J$-dependence for wide- $E_{\mathrm{g}}$ QDs $\left(E_{\text {emission }}=2.0\right.$ and $\left.2.2 \mathrm{eV}\right)$, while a linear correlation persists in the measured current density range for narrow- $E_{\mathrm{g}}$ QDs $\left(E_{\text {emission }}=1.7 \mathrm{eV}\right)$ (Fig. $\left.4 \mathrm{~b}\right)$. Interestingly, in the case of the
TCTA-based devices, no such a transition is observed and the linear dependence prevails regardless of $E_{\mathrm{g}}$ of QDs (Fig. 4c). Now that the super-linear relationship is associated with the intervention of the trap-involved nonradiative pathway (Supplementary Fig. 7b), varied slopes of the EL intensity- $J$ for different $E_{\mathrm{g}}$ result from the varying carrier injection rate into different QDs. In other words, narrow- $E_{\mathrm{g}}$ QDs primarily consume holes via exciton recombination although a larger number of holes at the QD-2TNATA interface may cause trap recombination for all QDs. This bias dependence is rather counter-intuitive in a model where the macroscopic electrostatic potential allows for initiating hole injection into $1 S_{h}$ states of all QDs owing to the minimal energy difference in $1 S_{h}$. And Augerassisted carrier injection mechanism ${ }^{5,35}$ cannot rationalize our observation because the up-conversion energy $\left(E_{\mathrm{CBM}}, \mathrm{ZnO}-\right.$ $\left.E_{\text {номо, нтьs }}, 1.2-1.8 \mathrm{eV}\right)^{40}$ exceeds the $E_{\mathrm{g}}$ difference of QDs. Therefore, the $E_{\mathrm{g}}$-dependent EL intensity demands an explanation as to why hole injection is facilitated in narrow- $E_{\mathrm{g}}$ QDs.

In the process of correlated charge injection ${ }^{36}$, an electron is injected into emissive materials prior to hole injection, which 
provides local Coulombic attraction $(U)$ and consequent enhancement of hole injection rate. To fully deconstruct the carrier injection processes in this context, we made further analysis on the 2TNATA-based devices. At $V<V_{\mathrm{L}}$, narrow- $E_{\mathrm{g}} \mathrm{QDs}$ are charged with an electron and wide- $E_{\mathrm{g}} \mathrm{QDs}$ are neutral [(i) and (ii) in Fig. 4e, respectively]. The electron in a narrow- $E_{\mathrm{g}} \mathrm{QD}$ alters the $1 \mathrm{~S}_{\mathrm{h}}$ state by $U=-0.24 \mathrm{eV}$ for given core size ${ }^{41}$ (Fig. $4 \mathrm{e}$ and Supplementary Note 4), which facilitates the hole injection by lowering $E_{1 \mathrm{~S}_{\mathrm{h}}}-\mathrm{E}_{\mathrm{VBM}}$, HTLs $\left[(\mathrm{i})\right.$ in Fig. 4e]. At bias $V \geq V_{\mathrm{L}}$, this local potential gain promotes a formation of neutral exciton and results in the linearity in EL intensity- $J$ curve (Fig. $4 \mathrm{~b}$ ). On the other hand, the large electron injection barrier $(\sim 0.5 \mathrm{eV})$ in the neutral wide- $E_{\mathrm{g}}$ QDs barely allows electrons from $\mathrm{ZnO}$ into the $1 \mathrm{~S}_{\mathrm{e}}$ states at $V \approx V_{\mathrm{L}}$. Without Coulombic assistance, the hole injection in $1 S_{\mathrm{h}}$ states would compete with the trap-involved process [(ii) in Fig. 4e]. The super-linear regime of wide- $E_{\mathrm{g}}$ QDs in the EL intensity- $J$ suggests the dominance of hole consumption in trap-assisted recombination (Fig. 4b). The electron injection of wide- $E_{\mathrm{g}}$ QDs is accelerated by the Coulombic attraction of the hole in a $1 S_{h}$ state at a higher electrical bias, where the slope in EL intensity- $J$ curve changes from 2.5 to 1 . On the other hand, in the case of TCTA-based QLEDs, the injection of both carriers takes place under sufficient bias $\left(V_{\mathrm{L}}\right.$, тCTA $=1.9$ $\mathrm{V})$. At $V \geq V_{\mathrm{L}}$, electrons introduced in overall QDs attract the holes and vice versa, enabling all QDs to contribute to the EL and yielding the linearity in the EL intensity-J, as shown in Fig. 4c. Low hole concentration at the QD-TCTA junction leads to fewer trap-involved recombination events.

In this sense, our interpretation addresses the discrepancy raised in the continuum DOS model for 2TNATA-based devices. The narrow- $E_{\mathrm{g}}$ QDs give off EL as the correlated, rapid injection of a hole outpaces the trap-assisted recombination, while the EL from wide- $E_{\mathrm{g}}$ QDs is suppressed due to the trap recombination pathway. As a result, the EL spectrum is skewed to exhibit disproportionately high emission intensity at lower end of the energy even at an elevated bias.

Our findings provide a window with which to view the operation of hybrid QLEDs from both macroscopic and microscopic perspectives. Of particular interest is the condition in which barrierless, balanced carrier injection can take place. From the macroscopic viewpoint, the core/shell QDs act as a class of semiconductors and modify the band structure of surrounding CTLs along the surface state-pinned $\mathrm{E}_{\mathrm{F}}$. The resulting electrostatic potential gain in the hybrid QLEDs is the main driving force for barrierless hole injection. From the microscopic perspective, the charge injected into individual QDs provides marginal local electrostatic potential gain for the opposite charge via Coulombic interaction. When only the injection barrier exceeds the extent of Coulombic attraction, the discrete nature of QDs becomes significant, only to cause uneven carrier injection, hence poor EL performance of QLEDs (as demonstrated in the 2TNATA device; see Supplementary Fig. 8). For the efficient operation of QLEDs, therefore, the injection barrier should be kept lower than the extent of Coulombic interaction. For example, our explanation offers hindsight as to recent highperformance InP QLEDs ${ }^{3}$ using ZnMgO ETLs with reduced electron affinity. We envision rigorous and thorough study on surface modification, e.g., via ligand exchange, as a definite must in the way of taking the efficiency and stability of QLEDs up a notch.

\section{Methods}

Materials. Indium acetate $\left(\mathrm{In}(\mathrm{Ac})_{3}, 99.99 \%\right)$, zinc acetate $\left(\mathrm{Zn}(\mathrm{Ac})_{2}, 99.99 \%\right)$, tris(trimethylsilyl)-phosphine ((TMS) $\left.{ }_{3} \mathrm{P}, 99.9 \%\right)$, 1-octadecene (ODE, 99\%), oleic acid (OA, 99\%), and tri-n-octylphosphine (TOP, 99\%) were purchased from UniAm. Selenium (Se, 99.99\%), sulphur (S, 99.98\%), zinc acetate dihydrate
( $\left.\mathrm{Zn}(\mathrm{Ac}) \cdot 2 \mathrm{H}_{2} \mathrm{O}, 99 \%\right)$, potassium hydroxide (KOH, 99.99\%), 1-octanethiol (OT, 98.5\%), zinc chloride $\left(\mathrm{ZnCl}_{2}, 99.995 \%\right)$, oleylamine (OLA, 70\%), trioctylphosphine oxide (TOPO, 99\%), ethanolamine (EA, 99\%), and lithium triethylborohydride (LBH, $1 \mathrm{M}$ in THF) were purchased from Sigma Aldrich. All anhydrous organic solvents were purchased from Daejung (Korea) and used after $\mathrm{N}_{2}$ purging. Organic hole injection layers were purchased from Lumtec. Molybdenum trioxide $\left(\mathrm{MoO}_{3}\right.$ 99.999\%) and $\mathrm{Al}$ (99.999\%) were purchased from Alfa Aesar. Au (99.99\%) was purchased from iTASCO. Unless noted otherwise, chemicals were used as received without further purification.

Synthesis of InP/ZnSe/ZnS QDs. All precursor solutions were prepared under inert atmosphere. We prepared $0.5 \mathrm{M} \mathrm{In}(\mathrm{OA})_{3}$ by degassing a mixture of $20 \mathrm{mmol}$ of $\mathrm{In}(\mathrm{Ac})_{3}$ and $19.7 \mathrm{~mL}$ of $\mathrm{OA}$ in a round-bottom flask at $130^{\circ} \mathrm{C}$ for $3 \mathrm{~h}$ and subsequently diluting the mixture with $20.3 \mathrm{~mL}$ of ODE. We prepared $0.5 \mathrm{M}$ $\mathrm{Zn}(\mathrm{OA})_{2}$ by reacting $20 \mathrm{mmol}$ of $\mathrm{Zn}(\mathrm{Ac})_{2}$ with $13 \mathrm{~mL}$ of $\mathrm{OA}$ at $120^{\circ} \mathrm{C}$ under vacuum for $3 \mathrm{~h}$ and adding $27 \mathrm{~mL}$ of ODE. To prepare $2 \mathrm{M}$ TOPSe (or $2 \mathrm{M}$ TOPS), we mixed $20 \mathrm{mmol}$ of Se (or S) with $10 \mathrm{~mL}$ of TOP, and then heated the mixture at $180^{\circ} \mathrm{C}$ for $2 \mathrm{hrs}$. All precursor solutions were stored in a nitrogen-filled glove box prior to use.

We synthesized InP cores by following a previously reported recipe ${ }^{15}$. In a three-neck round bottom flask, $20 \mathrm{~mL}$ of $0.5 \mathrm{M} \mathrm{In}(\mathrm{OA})_{3}$ and $150 \mathrm{~mL}$ of ODE were loaded and degassed at $110^{\circ} \mathrm{C}$ for $2 \mathrm{~h}$. After the reaction vessel was filled with $\mathrm{N}_{2}$, we injected a mixture of $10 \mathrm{~mL}$ of $0.5 \mathrm{M}$ (TMS) ${ }_{3} \mathrm{P}$ in TOP into the reaction flask, which was then heated up to $260^{\circ} \mathrm{C}$ and kept for $10 \mathrm{~min}$. To obtain $\mathrm{InP}$ cores with a mean diameter of $3.4 \mathrm{~nm}$, a mixture of $15 \mathrm{~mL}$ of $0.5 \mathrm{M} \mathrm{In}(\mathrm{OA})_{3}$ and $15 \mathrm{~mL}$ of $0.5 \mathrm{M}$ (TMS) ${ }_{3} \mathrm{P}$ was slowly added until the first exciton peak reaches ca. $570 \mathrm{~nm}$. After quenching the reaction mixture, we purified the crude solution twice by the precipitation (acetone)/redispersion (toluene) protocol. The resultant InP cores were dissolved in hexane (concentration: $100 \mathrm{mg} \mathrm{mL}^{-1}$ ) and stored in a glove box for further use.

For the $\mathrm{ZnSe} / \mathrm{ZnS}$ shell growth, $4 \mathrm{~mL}$ of $0.5 \mathrm{M} \mathrm{Zn}(\mathrm{OA})_{2}$ and $10 \mathrm{~mL}$ of ODE were degassed at $110^{\circ} \mathrm{C}$ for $2 \mathrm{~h}$. After back-filling with $\mathrm{N}_{2}, 0.5 \mathrm{~mL}$ of as-prepared InP core dispersion was injected to the flask at $180^{\circ} \mathrm{C}$ and aged for $30 \mathrm{~min}$. At $300^{\circ} \mathrm{C}, 1.0 \mathrm{~mL}$ of TOPSe was introduced dropwisely and kept for an hour to grow $2 \mathrm{~nm}$-thick ZnSe shell. To complete with $1 \mathrm{~nm}$-thick $\mathrm{ZnS}$ shell, $8 \mathrm{~mL}$ of $0.5 \mathrm{M}$ $\mathrm{Zn}(\mathrm{OA})_{2}$ and $2 \mathrm{~mL}$ of $2 \mathrm{M}$ TOPS were added to the reactor and waited for an hour. After the reaction is terminated, we purified the crude solution once using acetone and toluene. And added $0.1 \mathrm{~mL}$ of OT to improve colloidal stability during extensive purification. After purifying OT-treated QDs four times, the resulting QD dispersion in $\mathrm{n}$-octane was stored in a glove box for device fabrication.

Synthesis of ZnS NCs. We synthesized ZnS NCs via a previously reported synthetic protocol with slight modification ${ }^{42}$. Briefly, $8 \mathrm{mmol}$ of $\mathrm{ZnCl}_{2}, 40 \mathrm{~mL}$ of OLA and $9.2 \mathrm{~g}$ of TOPO were degassed at $150{ }^{\circ} \mathrm{C}$ for $2 \mathrm{~h}$. Separately, $36 \mathrm{mmol}$ of S and $15 \mathrm{~mL}$ of $\mathrm{OLA}$ were reacted at $150^{\circ} \mathrm{C}$ for $1 \mathrm{~h}$ under vacuum. After quenching precursor solutions, $2.5 \mathrm{~mL}$ of $\mathrm{S}$ precursor solution was added to the $\mathrm{Zn}$ precursor solution and was heated to $320^{\circ} \mathrm{C}$. After an hour, the crude solution was quenched to room temperature and purified using acetone and toluene twice. To coincide the surface chemistry of ZnS NCs' with that of QDs, as-prepared QDs were treated with $\mathrm{Zn}(\mathrm{OA})_{2}$ and TOPS, precursors used for the outmost $\mathrm{ZnS}$ shell. The overall $\mathrm{ZnS}$ NCs were mixed with $8 \mathrm{~mL}$ of $0.5 \mathrm{M} \mathrm{Zn}(\mathrm{OA})_{2}$ and $40 \mathrm{~mL}$ of ODE and degassed at $120^{\circ} \mathrm{C}$ for $2 \mathrm{~h}$. After back-filling with $\mathrm{N}_{2}, 2 \mathrm{~mL}$ of $2 \mathrm{M}$ TOPS was injected into the solution at $300^{\circ} \mathrm{C}$ and rested for an hour, which yields a mean diameter of $8.4 \mathrm{~nm}$. We purified the final product once with acetone and toluene, and treated with OT, as described above. The purified ZnS NCs were dissolved in $n$-octane and stored in the glove box.

Synthesis of ZnO NPs. We prepared ZnO NPs using a recipe published by Kwak et al. ${ }^{6}$ with minor modification. $2.5 \mathrm{~g}$ of $\mathrm{Zn}(\mathrm{Ac})_{2} \cdot 2 \mathrm{H}_{2} \mathrm{O}$ was dissolved in $100 \mathrm{~mL}$ of methanol at $60^{\circ} \mathrm{C}$ for $30 \mathrm{~min} .1 .25 \mathrm{~g}$ of $\mathrm{KOH}$ dissolved in $50 \mathrm{~mL}$ of methanol was separately prepared and added to the $\mathrm{Zn}(\mathrm{Ac})_{2} \cdot 2 \mathrm{H}_{2} \mathrm{O}$ solution at $60^{\circ} \mathrm{C}$. After $150 \mathrm{~min}$, white precipitate was collected by centrifugation and washed with methanol twice. To improve colloidal stability, the product was treated with $7 \mathrm{~mL}$ of ethanolamine solution ( $10 \mathrm{vol} \%$ in methanol) for $5 \mathrm{~min}$. To remove excess ethanolamine and adjust the concentration of the dispersion of $\mathrm{ZnO} \mathrm{NPs}$, we precipitated the product using a mixture of methanol, hexane, and isopropanol $(=1: 6: 1$ in volume ratio) and redispersed in desired amount of ethanol.

Characterization of InP/ZnSe/ZnS QDs and ZnS NCs. We obtained TEM images using an FEI Tecnai F20 electron microscope. Absorption and PL spectra were acquired using a Shimadzu UV-3600 spectrometer and a HORIBA Fluoromax-4 spectrometer. We measured absolute PL QY using a Hamamatsu, Quantaurus-QY C11347 spectrometer equipped with an integrating sphere, by exciting the samples at the wavelength of $450 \mathrm{~nm}$. PL QY of QDs is $72 \%$ in solution and $41 \%$ in film on the glass.

Fabrication of QLEDs. Indium-tin-oxide (ITO) substrates were washed with DI water, acetone, IPA and dried in a convection oven at $120^{\circ} \mathrm{C}$. ZnO ETL was deposited by spinning $20 \mathrm{mg} \mathrm{mL}^{-1}$ of $\mathrm{ZnO}$ solution on top of an ITO substrate at 
$4000 \mathrm{rpm}$ for $30 \mathrm{~s}$ and dried at $180^{\circ} \mathrm{C}$ for $30 \mathrm{~min}$, to yield a layer of about $30 \mathrm{~nm}$ thickness. QD EMLs with a thickness of $18 \mathrm{~nm}$ was deposited by spun the QD dispersion on the ITO/ZnO substrates in the same way above. We then transferred $\mathrm{ITO} / \mathrm{ZnO} / \mathrm{QD}$ substrates to a thermal evaporator, in which, organic HTLs $(60 \mathrm{~nm})$, $\mathrm{MoO}_{x}(10 \mathrm{~nm})$, and $\mathrm{Al}(100 \mathrm{~nm})$ were sequentially deposited at $1 \times 10^{6}$ Torr on the substrates with an evaporation rate of $1.0,0.2-0.3$, and $2.0 \AA \mathrm{s}^{-1}$, respectively. The QLEDs were encapsulated with UV-curable resin and kept in a glove box before use. For the ZnS-employed device, the $18 \mathrm{~nm}$ of $\mathrm{ZnS} \mathrm{NC}$ film was deposited likewise, where ZnS NCs simple replace QDs in the EMLs. We notify that the thin film fabrication and sample handling were thoroughly performed in $\mathrm{N}_{2}$ atmosphere $\left(\mathrm{O}_{2}\right.$ and $\left.\mathrm{H}_{2} \mathrm{O}<0.1 \mathrm{ppm}\right)$.

Device characterization. $J-V-L$ characteristics and power spectral density of EL were collected using a Konica Minolta CS-2000 spectroradiometer coupled with a Keithley 2400 source meter. $V_{\mathrm{J}}$ is determined by a voltage where $J$ is transformed from Ohmic current to Trap-filled current. $V_{\mathrm{L}}$ is a voltage where the light output from the device exceeds $1 \times 10^{-3} \mathrm{~cd} \mathrm{~m}^{-2}$, the detection limit of the spectroradiometer. Spectrally resolved EL intensity can be directly acquired from the power spectral density of EL at $1.7,2.0$, and $2.2 \mathrm{eV}$ for each data point. The capacitance was measured using an Ivium Technologies Vertex potentiostat by sweeping DC voltage with sinusoidal AC frequency of $100 \mathrm{~Hz}$ and amplitude of $0.1 \mathrm{~V}$.

Time-resolved PL analysis. Decay dynamics of global PL spectra were obtained using a Picoquant time-correlated single-photon counting system (TCSPC) which employed a pulsed laser diode at $488 \mathrm{~nm}$ and single-photon avalanche diodes acquiring photons from $500 \mathrm{~nm}$ to $1100 \mathrm{~nm}$. The excitation pulse had a resolution of $200 \mathrm{ps}$ and a repetition rate of $500 \mathrm{kHz}$. This low-energy excitation allowed for probing decay dynamics of QDs in ITO/ZnO/QD and ITO/ZnO/QD/HTLs samples without the participation of carriers generated in HTLs (Fig. 2c, f).

The spectrally-resolved PL decay of ITO/ZnO/QDs (Fig. 4a) was performed using a Horiba DeltaTime TCSPC kit, where the samples were excited at $402 \mathrm{~nm}$ using a laser diode with a resolution of $200 \mathrm{ps}$ and a repetition rate of $1 \mathrm{MHz}$. At the emission slit, signals were collimated by a monochromator with a bandpass of $3 \mathrm{~nm}$ and detected by a photomultiplier tube.

Negative trion decay of InP/ZnSe/ZnS QDs was characterized using LBH as an electrochemical dopant ${ }^{23}$. Briefly, in presence of LBH in the QD dispersion, ultraviolet light at $365 \mathrm{~nm}$ was illuminated for several minutes to cause photodoping. To prevent the formation of doubly charged QDs, average electron occupancy was limited to less than 0.3. A PL decay trace of neutralized QDs was acquired by exposing QD dispersion to air. By subtracting tail-normalized PL decay traces of charged and neutral QDs, we obtained negative trion decay dynamics with $\tau_{\mathrm{X}^{-}}=0.9 \mathrm{~ns}$, as shown in Supplementary Fig. 5 .

PL decay lifetimes and normalized pre-exponential factors for $\mathrm{X}, \mathrm{X}^{-}$, and ET are quantified using a tri-exponential decay model:

$$
y=\sum_{\substack{i=\\ \mathrm{X}, \mathrm{X}-\text { and } \mathrm{ET}}} A_{j} e^{-t / \tau_{i}}
$$

where $A_{\mathrm{i}}$ is a pre-exponential factor of $i$ and is relevant to the contribution of $i$ to the entire decay process, $\tau_{\mathrm{i}}$ is a recombination lifetime of $i, t$ is time, and $i$ is a decay channel of $\mathrm{X}, \mathrm{X}^{-}$, and ET. For convenience, we normalized the amplitude of PL intensity to 1 to establish $\sum A_{\mathrm{i}}=1$. Assuming that $\tau_{\mathrm{X}}$ and $\tau_{\mathrm{X}^{-}}$of QD films are identical to those of QD in solution and $\tau_{\mathrm{X}}=29 \mathrm{~ns}$ and $\tau_{\mathrm{X}^{-}}=0.9 \mathrm{~ns}$, we obtained $\tau_{\mathrm{ET}}, A_{\mathrm{X}}, A_{\mathrm{X}^{-}}$, and $A_{\mathrm{ET}}$ with high credibility of fit (See Supplementary Table 2). $\mathrm{A}_{\mathrm{X}^{-}}$can be considered as $f_{\mathrm{e}}$.

Ultraviolet photoelectron spectroscopy. ITO substrate was prepared by the washing protocol for QLEDs. A $150 \mathrm{~nm}$-thick Au-coated Si substrate was prepared by thermal evaporation of Au at $1 \times 10^{-6}$ Torr with an evaporation rate of $1.0 \mathrm{~A} \mathrm{~s}^{-1}$. ZnO, QDs, and ZnS NCs thin films were deposited on the substrates by spinning each dispersion at 4,000 rpm for $30 \mathrm{~s}$ in an $\mathrm{N}_{2}$-filled glove box. To investigate thickness-dependent energy level modification of HTLs, we thermally evaporated 5, 10, and $20 \mathrm{~nm}$-thick HTL thin films on top of ITO/ZnO/QD substrates with an evaporation rate of $1.0 \AA \mathrm{s}^{-1}$. To avoid surface contamination or degradation, the entire preparation and handling off all samples took place in $\mathrm{N}_{2}$ atmosphere $\left(\mathrm{O}_{2}\right.$ and $\left.\mathrm{H}_{2} \mathrm{O}<0.1 \mathrm{ppm}\right)$ until the sample was directly loaded to the ultra-high vacuum chamber for the UPS analysis. The UPS spectra were taken using Thermo VG Scientific Sigma Probe with He I light source $(h v=21.2 \mathrm{eV})$. The Fermi levels of samples were calibrated with respect to that of Au reference. We determined the cutoff energy ( $E_{\text {cutoff }}$ ) by taking linear extrapolation at the high binding energy region, where the work function $=21.2-E_{\text {cutoff. }} E_{\mathrm{F}}-E_{\mathrm{VBM}}$ was estimated using the onset energy $\left(E_{\text {onset }}\right)$ at low binding energy region and the ionization energy was computed from $\left(E_{\mathrm{He} \mathrm{I}}-\mathrm{E}_{\text {cutoff }}\right)+E_{\text {onset. }} E_{\mathrm{VBM}}-E_{\mathrm{g}}$ yields $E_{\mathrm{CBM}}$.

\section{Data availability}

The data that support the findings of this study are available from the corresponding author upon reasonable request.
Received: 22 April 2021; Accepted: 9 September 2021; Published online: 27 September 2021

\section{References}

1. Dai, X. et al. Solution-processed, high-performance light-emitting diodes based on quantum dots. Nature 515, 96-99 (2014).

2. Kim, T. et al. Efficient and stable blue quantum dot light-emitting diode. Nature 586, 385-389 (2020).

3. Won, Y. et al. Highly efficient and stable InP/ZnSe/ZnS quantum dot lightemitting diodes. Nature 575, 634-638 (2019).

4. Stouwdam, J. W. \& Janssen, R. A. J. Red, green, and blue quantum dot LEDs with solution processable $\mathrm{ZnO}$ nanocrystal electron injection layers. J. Mater. Chem. 18, 1889-1894 (2008).

5. Mashford, B. S. et al. High-efficiency quantum-dot light-emitting devices with enhanced charge injection. Nat. Photonics 7, 407-412 (2013).

6. Kwak, J. et al. Bright and efficient full-color colloidal quantum dot lightemitting diodes using an inverted device structure. Nano Lett. 12, 2362-2366 (2012).

7. Xu, T. et al. Highly simplified reddish orange phosphorescent organic lightemitting diodes incorporating a novel carrier-and exciton-confining spiroexciplex-forming host for reduced efficiency roll-off. ACS Appl. Mater. Interfaces 9, 2701-2710 (2017).

8. Lim, J., Park, Y. S., Wu, K., Yun, H. J. \& Klimov, V. I. Droop-free colloidal quantum dot light-emitting diodes. Nano Lett. 18, 6645-6653 (2018).

9. Bae, W. K. et al. Controlling the influence of Auger recombination on the performance of quantum-dot light-emitting diodes. Nat. Commun. 4, 2661 (2013).

10. Schubert, E. F. Light-Emitting Diodes. (Cambridge University Press, 2006).

11. Qian, L., Zheng, Y., Xue, J. \& Holloway, P. H. Stable and efficient quantumdot light-emitting diodes based on solution-processed multilayer structures. Nat. Photonics 5, 543-548 (2011).

12. Luo, H. et al. Origin of subthreshold turn-on in quantum-dot light-emitting diodes. ACS Nano 13, 8229-8236 (2019).

13. Yang, Y. et al. High-efficiency light-emitting devices based on quantum dots with tailored nanostructures. Nat. Photonics 9, 259-265 (2015).

14. Acharya, K. P. et al. High efficiency quantum dot light emitting diodes from positive aging. Nanoscale 9, 14451-14457 (2017).

15. Hahm, D. et al. Design principle for bright, robust, and color-pure InP/ ZnSexS1-x/ZnS heterostructures. Chem. Mater. 31, 3476-3484 (2019).

16. Xiang, C. et al. High efficiency and stability of ink-jet printed quantum dot light emitting diodes. Nat. Commun. 11, 1646 (2020).

17. Klimov, V. I., McGuire, J. A., Schaller, R. D. \& Rupasov, V. I. Scaling of multiexciton lifetimes in semiconductor nanocrystals. Phys. Rev. B 77, 195324 (2008).

18. Horowitz, G., Delannoy, P., Horowitz, G. \& Delannoy, P. An analytical model for organic-based thin-film transistors. J. Appl. Phys. 70, 469-475 (1991).

19. Biswas, S., Kar, S. \& Chaudhuri, S. Optical and magnetic properties of manganese-incorporated zinc sulfide nanorods synthesized by a solvothermal process. J. Phys. Chem. B 109, 17526-17530 (2005).

20. Wang, X., Shi, J., Feng, Z., Li, M. \& Li, C. Visible emission characteristics from different defects of ZnS nanocrystals. Phys. Chem. Chem. Phys. 13, 4715-4723 (2011).

21. Anderson, N. C., Hendricks, M. P., Choi, J. J. \& Owen, J. S. Ligand exchange and the stoichiometry of metal chalcogenide nanocrystals: Spectroscopic observation of facile metal-carboxylate displacement and binding. J. Am. Chem. Soc. 135, 18536-18548 (2013).

22. Janotti, A. \& de Van Walle, C. G. Fundamentals of zinc oxide as a semiconductor. Rep. Prog. Phys. 72, 126501 (2009).

23. Rinehart, J. D., Schimpf, A. M., Weaver, A. L., Cohn, A. W. \& Gamelin, D. R. Photochemical electronic doping of colloidal CdSe nanocrystals. J. Am. Chem. Soc. 135, 18782-18785 (2013).

24. Somorjai, G. A. \& Li, Y. Introduction to Surface Chemistry and Catalysis. (John Wiley \& Sons, 2010).

25. Zandi, O. et al. Impacts of surface depletion on the plasmonic properties of doped semiconductor nanocrystals. Nat. Mater. 17, 710-717 (2018).

26. Jäger, L., Schmidt, T. D. \& Brütting, W. Manipulation and control of the interfacial polarization in organic light-emitting diodes by dipolar doping. AIP $A d v .6,095220$ (2016)

27. Wang, $\mathrm{X}$. et al. Amorphous $\mathrm{ZnO} / \mathrm{PbS}$ quantum dots heterojunction for efficient responsivity broadband photodetectors. ACS Appl. Mater. Interfaces 12, 8403-8410 (2020).

28. Sun, F. Z. et al. Efficient inverted polymer solar cells with thermal-evaporated and solution-processed small molecular electron extraction layer. Appl. Phys. Lett. 102, 133303 (2013). 
29. Park, J. W. et al. Electron-injecting properties of Rb2CO3-doped Alq3 thin films in organic light-emitting diodes. J. Vac. Sci. Technol. A 31, 031101 (2013).

30. Ji, W. et al. Ultrasonic spray processed, highly efficient all-inorganic quantumdot light-emitting diodes. ACS Photonics 4, 1271-1278 (2017).

31. Okamura, T., Seki, Y., Nagakari, S. \& Okushi, H. Preparation of n-ZnO/p-Si heterojunction by sol-gel process. Jpn. J. Appl. Phys. 31, L762 (1992).

32. Nowy, S., Ren, W., Elschner, A., Lövenich, W. \& Brütting, W. Impedance spectroscopy as a probe for the degradation of organic light-emitting diodes. $J$. Appl. Phys. 107, 054501 (2010).

33. Mártil, I., Redondo, E. \& Ojeda, A. Influence of defects on the electrical and optical characteristics of blue light-emitting diodes based on III-V nitrides. J. Appl. Phys. 81, 2442-2444 (1997).

34. Blood, P. Quantum Confined Laser Devices: Optical gain and Recombination in Semiconductors. (OUP Oxford, 2015).

35. Qian, L. et al. Electroluminescence from light-emitting polymer/ZnO nanoparticle heterojunctions at sub-bandgap voltages. Nano Today 5, 384-389 (2010).

36. Lim, J., Park, Y. S. \& Klimov, V. I. Optical gain in colloidal quantum dots achieved with direct-current electrical pumping. Nat. Mater. 17, 42-48 (2018).

37. Shen, H. et al. Visible quantum dot light-emitting diodes with simultaneous high brightness and efficiency. Nat. Photonics 13, 192-197 (2019).

38. Crooker, S. A., Hollingsworth, J. A., Tretiak, S. \& Klimov, V. I. Spectrally resolved dynamics of energy transfer in quantum-dot assemblies: towards engineered energy flows in artificial materials. Phys. Rev. Lett. 89, 186802 (2002).

39. Vurgaftman, I., Meyer, J. R. \& Ram-Mohan, L. R. Band parameters for III-V compound semiconductors and their alloys. J. Appl. Phys. 89, 5815-5875 (2001).

40. He, S.-J., Wang, D.-K., Jiang, N., Zhang, J. \& Lu, Z.-H. Auger-electronstimulated organic electroluminescence at ultralow voltages below the energy gap. Phys. Rev. Appl. 3, 054011 (2015).

41. Lannoo, M., Delerue, C. \& Allan, G. Screening in semiconductor nanocrystallites and its consequences for porous silicon. Phys. Rev. Lett. 74, 3415-3418 (1995).

42. Joo, J. et al. Generalized and facile synthesis of semiconducting metal sulfide nanocrystals. J. Am. Chem. Soc. 125, 11100-11105 (2003).

\section{Acknowledgements}

D.C.L. and H.L. gratefully acknowledge support from the National Research Foundation of Korea (NRF) grants funded by the Korean Government (NRF-2016M3A7B4910618, NRF2021M3H4A1A01004332, and NRF-2020M3D1A2101310) and the Ministry of Trade, Industry \& Energy (MOTIE, Korea) under Industrial Strategic Technology Development Program (No. 10077471). D.C.L., J.L., and W.K.B. acknowledge the support from the NRF grant funded by the Korean Government (NRF-2021M3H4A3A01062960). J.L. and H.L. acknowledge support from the NRF grants funded by the Ministry of Science and ICT (NRF2019R1C1C1006481 and NRF-2019M3D1A1078299) and the Samsung Research Funding \& Incubation Centre of Samsung Electronics under Project Number SRFC-TB1903-02.

\section{Author contributions}

H.L. designed and analyzed the QLEDs and prepared the paper. B.G.J. and W.K.B. synthesized the QDs. D.C.L. and J.L. initiated the studies and supervised experiments, analysis, and paper preparation.

\section{Competing interests}

The authors declare no competing interests.

\section{Additional information}

Supplementary information The online version contains supplementary material available at https://doi.org/10.1038/s41467-021-25955-z.

Correspondence and requests for materials should be addressed to Doh C. Lee or Jaehoon Lim.

Peer review information Nature Communications thanks the anonymous reviewer(s) for their contribution to the peer review of this work.

Reprints and permission information is available at http://www.nature.com/reprints

Publisher's note Springer Nature remains neutral with regard to jurisdictional claims in published maps and institutional affiliations.

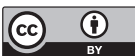

Open Access This article is licensed under a Creative Commons Attribution 4.0 International License, which permits use, sharing, adaptation, distribution and reproduction in any medium or format, as long as you give appropriate credit to the original author(s) and the source, provide a link to the Creative Commons license, and indicate if changes were made. The images or other third party material in this article are included in the article's Creative Commons license, unless indicated otherwise in a credit line to the material. If material is not included in the article's Creative Commons license and your intended use is not permitted by statutory regulation or exceeds the permitted use, you will need to obtain permission directly from the copyright holder. To view a copy of this license, visit http://creativecommons.org/ licenses/by/4.0/.

(C) The Author(s) 2021 